\title{
NÚMEROS NATURALES: DISTINTAS METODOLOGÍAS QUE CONVERGEN EN EL ANÁLISIS DE SU NATURALEZA Y DE CÓMO LOS ENTENDEMOS
}

José Ferreirós y Abel Lasalle Casanave (coordinadores), El árbol de los números: cognición, lógica y práctica matemática, Editorial Universidad de Sevilla, Sevilla, 2015, 256 pp.

Melisa Vivanco

University of Miami mxg1494@miami.edu

\section{Sobre la compilación}

En torno al debate sobre la existencia de una realidad matemática, a la par de una (más o menos bien aceptada) realidad física, tradicionalmente se han planteado diversas preguntas de interés filosófico. Entre las más clásicas está, por ejemplo, la cuestión de cómo obtenemos conocimiento acerca de los objetos que presuntamente habitarían dicha realidad. Desde una postura realista, tener conocimiento de la realidad matemática no es sino tener conocimiento de dichos objetos y su comportamiento. Sin embargo, como ha sido ampliamente discutido (Bueno 2017; Field 1980, 1989; Yablo 2010, 2014; etc.), negar la existencia de dichos objetos no excluye el reconocimiento del conocimiento matemático. En particular, hablando de la aritmética, negar la existencia de los números no excluye la posibilidad del conocimiento aritmético. Lo que es relativamente claro es que, desde cualquier tipo de postura ontológica acerca de los números, es necesario explicar la naturaleza de aquello que identificamos como conocimiento aritmético. Otra cuestión íntimamente relacionada con la postura ontológica es el tipo de teoría acerca de la verdad aritmética que puede ser aceptada. Aquí cabe señalar la distinción entre un realismo ontológico, acerca de los números de los que hablan los enunciados que contienen términos numéricos, y un realismo en valor de verdad. Este último afirma la existencia de ciertos hechos, posiblemente independientes de la existencia de las entidades que vendrían siendo los referentes de los términos numéricos, que hacen verdaderos a los enunciados de la aritmética pura, de otras áreas de la matemática 
y, en último término, de cualquier enunciado con términos numéricos en el lenguaje natural. Finalmente, vale la pena mencionar que cualquier teoría filosófica en este ámbito deberá dar cuenta de la característica propiedad que tiene la aritmética de generar resultados exitosos en sus aplicaciones, que van desde los contextos más formales, pasando por contextos predominantemente técnicos, hasta llegar a los más simples contextos cotidianos.

Teniendo en cuenta las cuestiones anteriormente mencionadas, entre muchas otras, José Ferreirós y Abel Lassalle Casanave nos presentan en su libro El árbol de los números una compilación de artículos que, además de interesante y novedosa, resulta altamente enriquecedora debido al enfoque plural que los compiladores logran a través de la organización de una selección de artículos que permite al lector tener una aproximación bastante comprehensiva a los debates actuales en filosofía de las matemáticas, específicamente a aquellos enfocados en los números naturales.

El lector o lectora podría sentir la tentación de preguntar cómo las teorías presentadas en El árbol de los números encajan en una explicación más amplia que abarque otras áreas de la matemática. Por esta razón, vale la pena mencionar que el hecho de que los artículos elegidos no vayan más allá de los números naturales no resta de ninguna manera el interés que cualquier filósofo de la matemática tendría en este trabajo. Por múltiples razones, los números naturales son un buen punto de partida para una investigación filosófica sobre la matemática. Entre las razones matemáticas, por ejemplo, tenemos que la estructura de los números naturales antecede a otras estructuras tanto en un sentido temporal, como desde la misma práctica de la disciplina. Como es bien sabido, la axiomatización de la aritmética en el siglo XIX fue el comienzo de un paradigma en la forma en la que se desarrolla la matemática. Respecto a la práctica, vale la pena tener en mente que la estructura de los números naturales se utiliza como base para la construcción formal de otros sistemas numéricos, que dan lugar a nuevas estructuras algebraicas. A partir de los números naturales se construyen los números enteros (ejemplo paradigmático de los anillos y los dominios enteros); los números racionales (ejemplo paradigmático de campo numerable); los números reales (ejemplo paradigmático de campo no numerable), etcétera.

Además de las razones matemáticas, existen razones filosóficas para priorizar las explicaciones acerca de los números naturales. Consideremos, por poner un ejemplo, una de las más clásicas preocupaciones de la filosofía - la preocupación acerca de la certeza-. Los enunciados 
aritméticos (aquéllos cuyos términos singulares refieren a números naturales) son típicamente considerados necesariamente verdaderos y cuyo contenido es conocido a priori. Cómo los enunciados aritméticos podrían gozar de este privilegio epistémico es, sin duda, de gran interés filosófico.

Una motivación adicional para desarrollar el tema de los números, que los compiladores no pasan de largo, es el propósito de dar cuenta de qué función tienen en las prácticas cotidianas y de cómo es la relación de los seres humanos con aquello que es concebido como los números desde la infancia misma. Las oraciones que contienen numerales aparecen en prácticamente cualquier parte del discurso humano. Es poco controversial que hay una relación entre la competencia lingüística y la competencia aritmética de un hablante común. Determinar cuál es esta relación es también interés de las ciencias cognitivas. De este modo, los estudios acerca de la adquisición de los números naturales y el desarrollo de habilidades cognitivas relacionadas con la aritmética responderán a importantes preguntas que tradicionalmente se han formulado en el campo de la cognición humana.

El interés de los compiladores se centra en las innovaciones de los últimos años en el análisis filosófico de la aritmética, en particular, atendiendo a la práctica matemática y teniendo en consideración las diversas contribuciones en el análisis cognitivo del número desde la neurociencia y las otras ciencias cognitivas. La selección posibilita una aproximación novedosa, que va más allá de la tradición inaugurada por filósofos y matemáticos como Frege, Dedekind, Peano y Russell, y que le permite al lector o lectora incorporarse a debates filosóficos actuales desde las perspectivas de la matemática misma y la neurociencia. En este sentido, el libro aborda el concepto de número desde tres flancos distintos pero complementarios: los fundamentos lógico-matemáticos, la práctica matemática y la ciencia cognitiva.

Una de las motivaciones que determina la forma en la que los compiladores seleccionaron y organizaron los trabajos está basada en una crítica respecto a cierta metodología, en la que predominó el interés por los fundamentos de la matemática a partir de la influencia de consideraciones surgidas desde aspectos concernientes a la lógica,

En buena medida, las dificultades filosóficas y fundacionales [...] provienen del énfasis excesivo que el siglo XX puso en visiones sistematizantes, omniabarcantes y reduccionistas. Durante las últimas décadas, el desarrollo de la llamada filosofía de las prácticas matemáticas ha venido a renovar y liberar el estudio de los problemas relacionados. (pp. 11, 12) 
Se podría pensar que esta crítica deja de lado la importancia histórica que las llamadas escuelas de los fundamentos tienen sobre la filosofía actual de las matemáticas. Los tratamientos filosófico-matemáticos de finales del siglo XIX y principios del XX dieron lugar a grandes avances no sólo para la filosofía, sino también para la matemática y la lógica. Sin embargo, la novedosa perspectiva establecida por los coordinadores, quienes (entre otras cosas) brindan una mayor contribución de los estudios empíricos relacionados con la adquisición de los números, amplía las perspectivas desde las cuales se pueden abordar los complejos problemas del conocimiento matemático, comenzando por el conocimiento aritmético.

Ciertamente, es indispensable para una teoría filosófica sobre la matemática que ésta sea compatible con la práctica de la disciplina. Pero tampoco debemos perder de vista la aproximación histórica, desde la que es fácil observar que la matemática y su filosofía no serían lo que son, de no ser por las inquietudes (calificadas por algunos, como fundacionistas) que condujeron a prácticas como la búsqueda de la axiomatización de áreas centrales en la matemática. Otro ejemplo de un avance que comprendemos mejor desde una aproximación histórica es el desarrollo de la teoría de conjuntos y posteriormente de la teoría de categorías, ampliamente motivado por la búsqueda de un lenguaje que permita la construcción de un marco que provea de cimientos teóricos a otras ramas de la matemática. Más ejemplos de los beneficios de estas tradiciones pueden ser localizados en la lógica y su filosofía, áreas que con frecuencia no carecen de algún interés matemático. Sin ir muy lejos, en estas tradiciones encontramos la semilla de importantes contribuciones, como la teoría de la demostración o el desarrollo de las lógicas no clásicas, teniendo como ejemplo paradigmático la lógica intuicionista y cierta práctica matemática basada en esta lógica. Yendo más hacia la filosofía de la lógica, pero sin abandonar las cuestiones matemáticas, tenemos que el histórico error de Frege, al postular la ley básica V como parte de los principios para fundamentar la aritmética desde su visión logicista (1884), ha dado lugar a importantes contribuciones a partir del análisis de la naturaleza de este tipo de principios (véase Hale y Wright 2009, para un ejemplo).

En conclusión, el éxito de una investigación profunda acerca de la naturaleza de los números y nuestro conocimiento de ellos dependerá de la capacidad que tengamos de integrar las diferentes perspectivas que se brindan, tanto desde un enfoque histórico que nos permita adentrarnos en la tradición de las escuelas de los fundamentos, como de un enfoque más actual; por ejemplo, aquellos que consideran 
la práctica matemática contemporánea y los datos empíricos sobre el fenómeno de adquisición. En este sentido, el libro presenta una importante contribución en el marco de una metodología actual, donde podría pensarse que se paga un pequeño costo respecto a la cuestión histórica de una metodología que, a pesar de tener sus defectos, fue altamente prolífica para la filosofía de la matemática, en particular, de la aritmética. Además de lo ya mencionado, el libro también conecta, en la línea de Lakatos y Kitcher, los aspectos filosóficos con el estudio de episodios de la práctica matemática, abriendo también el campo a problemas educativos de la aritmética.

El árbol de los números es un libro altamente recomendable para cualquier persona interesada en los debates contemporáneos sobre la naturaleza de los números, la filosofía de la práctica matemática (particularmente de la aritmética) y el conocimiento aritmético desde la aproximación de las ciencias cognitivas.

Lo anteriormente mencionado pretende sugerir un panorama respecto al espíritu de la compilación. Debido a que los artículos varían mucho en enfoques y posiciones, y cada lector o lectora podría tener intereses diferentes respecto a partes específicas de la compilación, en lo que sigue, se presentan breves reseñas de cada artículo con algunos comentarios.

\section{Parte I: cognición}

Los trabajos de la primera parte examinan diversos aspectos sobre la cognición matemática y sus bases neurológicas. En esta sección del libro se presentan trabajos de Giardino; Arrigoni y Caprile y Benis-Sinaceur. En estos trabajos se da un consenso respecto a que la cognición numérica tiene raíces innatas, que se constatan en estudios psicológicos y neurológicos. La idea es que existen dos núcleos cognitivos innatos "cableados" en zonas diferentes del cerebro: uno de ellos que permitiría la subitización (captación inmediata y precisa de números muy pequeños), mientras que el otro sería un sistema de aproximación de procesamiento de numerosidades pequeñas o grandes. Cuál es el papel que desempeñan estas habilidades, y cómo se relacionan entre sí, es algo sobre lo que cada teoría presentada en el libro acerca de la adquisición de los números enteros positivos tiene algo que decir. 
2.1. ¿Dónde situar los fundamentos cognitivos de las matemáticas? (Valeria Giardino)

El artículo se enfoca en la aportación de las ciencias cognitivas a la investigación acerca de los fundamentos (cognitivos) de las matemáticas. La tesis central es que, tanto para el caso de la aritmética como de la geometría, hay que distinguir entre tres niveles de competencia.

Competencia aritmética: en el nivel I se encuentra la capacidad de distinguir entre diferentes numerosidades. La matemática en este nivel es sólo aproximada. En el nivel II se encuentra la capacidad de contar. Contar como un proceso es hacer uso de los números en cuanto simples posiciones de una secuencia ordenada. En el nivel III está la aritmética teórica, los números se convierten en entidades abstractas y los que practican aritmética han desarrollado conceptos para ellos.

Competencia geométrica: en el nivel I se encuentra la capacidad de extraer invariantes del espacio circundante. Esta capacidad nos permite realizar tareas como aprender a orientarnos en un ambiente que hemos explorado anteriormente. En el nivel II nos encontramos con el uso de mapas y otras herramientas que nos ofrecen una representación externa sobre la cual nos apoyamos para orientarnos en un ambiente, ahorrándonos costos cognitivos. En el nivel III tenemos la geometría, las formas se han convertido en entidades abstractas, y se han desarrollado conceptos para ello.

El primer nivel está ligado a la percepción y el tercero a la competencia plena. La autora da una importancia especial al nivel intermedio. Este nivel, que involucra el uso de representaciones explícitas, es esencial para entender los fundamentos cognitivos de las matemáticas, debido a que somos la única especie simbólica capaz de manejar representaciones como los numerales, las cifras o los mapas. Para Giardino, esto es lo que, en última instancia, nos permite desarrollar conocimiento matemático.

Las distinciones que Valeria Giardino traza son por demás interesantes. Sin embargo, queda la cuestión de si este esquema realmente describe cómo son (o dónde se encuentran) los fundamentos cognitivos de las matemáticas. Para la autora, estos sistemas de representación son necesarios para lograr una forma de razonamiento abstracto (condición necesaria para la adquisición de conceptos matemáticos), pero de esto no se sigue que en el segundo nivel se encuentren los mencionados fundamentos. Para sostener su tesis, Giardino presenta experimentos con bebés, animales y poblaciones con pocas palabras, es decir, con individuos que cuentan con escasos recursos de repre- 
sentación. De acuerdo con su interpretación, estos sujetos de estudio no alcanzan nunca la posesión de los conceptos matemáticos porque carecen de las representaciones necesarias. En este punto valdría la pena preguntarse cuáles, específicamente, son esas representaciones. Sin embargo, teniendo en cuenta que los animales, los bebés y las comunidades con pocas palabras tienen acceso a herramientas de representación muy diferentes tanto en calidad como en cantidad, vale la pena preguntarse cuáles son las representaciones que resultarían suficientes para poder dar el salto hacia la adquisición de conceptos matemáticos. Se intuye que la respuesta estará relacionada con el lenguaje, pero si éste es el caso, pareciera haber un salto explicativo entre las habilidades lingüísticas y las capacidades geométricas.

La pregunta central de en dónde se encuentran los fundamentos en cuestión parece depender de la respuesta a una pregunta de la cual aún no es claro que tengamos el resultado. Ésta es, ¿cómo damos el salto desde el conocimiento de particulares, que adquirimos vía la percepción, a un conocimiento de tipo más general, que parecería requerir una cierta capacidad de abstracción? En caso de que el nivel II constituya este puente explicativo, habrá que hacer un estudio más detallado de su naturaleza y su relación con los otros dos niveles.

\subsection{La cognición de los enteros: una nueva propuesta (Tatiana Arrigoni y Bruno Caprile)}

Arrigoni y Caprile centran su atención en la comprensión - por parte del niño - de la infinitud de los números. La idea es que la cognición humana desarrolla lo que los autores llaman una teoría de los enteros como un género de objetos abstractos, en contraste con una comprensión adjetiva de los números como propiedades de colecciones de cosas. El análisis se enfoca en el estadio en torno a los 8 años, cuando los niños categorizan los números como un género de cosas con propiedades características, una de las cuales resultará ser su infinitud. Esto es, ser una sucesión ordenada literalmente interminable. De acuerdo con los autores, el objetivo es sugerir una nueva aproximación a la adquisición de los enteros positivos dirigida a determinar qué facultades independientes contribuyen a la misma.

El artículo sostiene que hay al menos dos teorías acerca de los enteros en los adultos:

a) Una teoría de los enteros como expresión de diferentes cardinalidades. Es decir, medidas exactas de multiplicidades de elementos 
identificables contando (esta teoría se refleja implícitamente en el uso lingüístico de los numerales como adjetivos); y

b) Una teoría de los enteros como objetos abstractos mutuamente relacionados, denotados o bien informalmente, en un lenguaje en el que los numerales desempeñan un papel de nombres, o (más o menos) formalmente usando un sistema específico de símbolos, como el sistema decimal posicional.

La interconexión de estas dos teorías parece ser típica de la cognición numérica de los humanos en sus formas más maduras. En contraste, los preescolares parecen poseer una teoría de los enteros como cardinalidades, que desarrollan mientras aprenden a contar, pero no una teoría de los enteros como objetos abstractos. Las cardinalidades son únicamente propiedades de multitudes de objetos espacio temporales. Los autores conjeturan que ésta podría ser la causa de que la teoría de los cardinales de los preescolares no dé información respecto a si los enteros son o no infinitos (más adelante, al adquirir la teoría b), los niños serán capaces de dar esta información). La pregunta obvia aquí es cómo se pasa de la competencia en una teoría del tipo a) hacia una del tipo b). (Un cuestionamiento similar surge para el caso de la explicación que Valeria Giardino ofrece en el artículo anterior.) La siguiente, pudiera ser la respuesta de los autores a la mencionada cuestión.

Se puede apartar a las cardinalidades del mundo espacio temporal, siendo vistas como medidas de la multiplicidad de cualquier tipo, incluyendo objetos imaginarios. Bajo estas circunstancias, apelando a nuestra capacidad para extender por medio de la imaginación cualquier multitud de objetos, y considerando la totalidad de las multitudes que pueden ser así generadas, podemos llegar a la infinitud de los enteros. (p. 54)

El problema es que esto requiere cierta capacidad mental, que no ha sido explicada: quizá una capacidad de abstracción lo bastante sofisticada para atribuirles propiedades a las entidades resultantes de este proceso. Sigue siendo crucial para todas estas teorías explicar de dónde proviene este tipo de habilidad. Para brindar una respuesta, los autores pueden apelar a que la ontogénesis de la cognición humana de los enteros desempeña un papel fundamental en la habilidad de los niños de: a) tratar a los enteros como objetos; b) hacer esto a pesar de que no tengan características espacio temporales; c) adscribirlos a un tipo determinado, independientemente de la disponibilidad de información estable explícita acerca de las propiedades que deberían 
compartir (para que pertenezcan al mismo tipo). De acuerdo con esto, los niños parecen darse cuenta de que los números son infinitos y de que cada uno de ellos tiene un sucesor, como hechos que pertenecen a un tipo determinado en el que comparten una naturaleza en común al que ellos se refieren con la expresión los números. Aun así, parece haber un hueco explicativo en lo que se refiere a la habilidad de los niños para hacer correctamente las adscripciones a los números como correspondiendo al tipo de entidades al que pertenecen. Presumiblemente, esta habilidad tendrá alguna especie de relación con las capacidades lingüísticas desarrolladas.

La observación final de los autores es que, el que los enteros sean abstractos y causalmente inertes para nosotros no es un motivo para evitar que los tratemos como objetos (lingüística y cognitivamente). En vez de negar el estatus de objeto a entidades no espacio temporales y causalmente inertes, a las que tratamos como tales, deberíamos extender nuestra noción de lo que cuenta como objeto para los humanos de manera que acomode también a entidades como los enteros que son, de facto, objetos para nosotros (lingüística y cognitivamente). Desde luego, esto es materia más de la metafísica que de las ciencias cognitivas (para una aproximación a este debate, véanse, por ejemplo, Balaguer 1998; Linnebo 2018).

\subsection{Filosofía de la biopsicología del número (Hourya}

Benis-Sinaceur)

Este artículo presenta una aproximación más técnica que la de los anteriores. En él se presentan resultados sobre las bases neurocientíficas de la captación de numerosidad. Estos resultados se toman de algunos artículos especializados y de tres renombrados libros en la materia (The Number Sense. How the Mind Creates Mathematics, de Stanislas Dehaene; Seeing and Visualizing, de Zenon Pylyshyn; y The Origin of Concepts de Susan Carey).

Benis-Sinaceur realiza una interpretación de los datos, resultado de diferentes experimentos, desde una aproximación de interés propiamente filosófico. De esta manera, la autora pretende relacionar los datos obtenidos en estudios neurobiológicos y psicológicos con preguntas como ¿Qué es anterior cognitivamente, lo continuo o lo discreto? ¿la cantidad o la cualidad? Respecto al sentido numérico y al contar efectivo, ¿̨cuál precede a cuál?

Típicamente, la pregunta de interés desde el punto de vista de las ciencias cognitivas es cómo captan el cerebro y la mente la dimensión cuantitativa de los eventos y fenómenos del mundo. Benis-Sinaceur 
señala - acertadamente, creo- que las respuestas al cómo no excluyen un replanteamiento de las concepciones sobre la naturaleza o el origen de los números. Pero en vista de que la autora pretende hacer una asociación entre los estudios en ciencias cognitivas y las cuestiones filosóficas señaladas, cabe mencionar que no es claro tampoco que dichas respuestas aporten evidencia para la aceptación de alguna concepción filosófica sobre los números. Muchos estudios en ciencias cognitivas han intentado encontrar en los datos empíricos resultados que apoyen resultados filosóficos. Pero ya sea para descartar posibilidades o para fortalecer tesis, siempre será necesario presentar un argumento filosófico en algún sentido plausible.

No es ningún misterio que los fenómenos neurobiológicos y psicológicos inducen cuestiones de carácter filosófico. La autora logra de manera clara señalar la existencia de algunas asociaciones entre los fenómenos empíricos y las concepciones filosóficas. Sin embargo, este hecho no parece ser realmente controversial. Lo que resultaría más interesante, sería señalar en dónde y cómo se dan dichas asociaciones. El artículo no parece ofrecer alguna propuesta positiva al respecto. Consideremos algunos ejemplos.

De acuerdo con Benis-Sinaceur, además de la división entre lo innato y lo adquirido, los test experimentales que implican cantidades o conjuntos de objetos suponen, o inducen, opciones relativas a las relaciones entre el contar y la evaluación de la cantidad; entre continuo y discreto; entre el aspecto ordinal y cardinal del número; entre percepción sensorial y concepto; entre percepción cualitativa y percepción cuantitativa; entre intuición e inferencia; entre imagen y pensamiento; entre pensamiento y lenguaje. La determinación de dichas relaciones en el seno de la anterioridad ontológica o cognitiva de cada una de estas parejas, como ejemplo de lo continuo sobre lo discreto, de la cualidad sobre la cantidad, y de la cantidad sobre contar, depende no sólo de la edad de los sujetos estudiados, de los tipos de cuestiones planteadas, de las técnicas de investigación privilegiadas y de sus diversas puestas en práctica, sino también de opciones filosóficas declaradas o tácitas que enmarcan la interpretación de las respuestas.

Por un lado, no resulta muy controvertida la afirmación de que los compromisos epistémicos de quien realiza los test experimentales influyen en la interpretación de los datos obtenidos (si acaso, habrá desacuerdo en la manera en la que se da esta influencia, o en qué grado). Por el otro lado, si, como dice la misma autora, los compromisos epistémicos interfieren en la interpretación de los datos, no 
podemos ver de forma inmediata cómo los estudios neurobiológicos y psicológicos aportan evidencia sustantiva (u objetiva) a las diferentes concepciones filosóficas. Sigue sin ser claro cómo se da la relación entre la evidencia empírica y las concepciones filosóficas.

La autora presenta los estudios como un material distribuido en modelos destinados a reformar nuestra visión filosófica sobre la naturaleza y origen de los números y nuestros principios educativos, para facilitar la adquisición de habilidades aritméticas. Su conclusión es que estos modelos son tributarios de puntos de vista correspondientes a una preferencia filosófica o a un estado del desarrollo de la ciencia y la cultura caracterizado por la abundante utilización de estructuras como medio de investigación, de organización y de análisis de los datos. Este resultado no debería generar mayor controversia. Lo interesante aquí sería tener un mapa que ejemplifique de manera clara las relaciones entre los estudios empíricos y el análisis filosófico.

\section{Parte II: lógica}

Los artículos compilados en la segunda parte del libro examinan diferentes aspectos de carácter lógico y matemático de los números enteros. Esta parte resulta interesante no sólo por el análisis filosófico y su énfasis en la adecuación de cada teoría respecto a un sistema lógico, sino también porque dibuja un panorama histórico desde el cuál se toman en consideración las aportaciones de filósofos y matemáticos de la talla de Frege, Dedekind, Peano, Russell, Whitehead y Gödel. En esta sección lógico-matemática se recopilan trabajos de Oswaldo Chateubriand, Concha Martínez Vidal, Thomas Sautter y Sérgio Shultz.

\subsection{Números como propiedades de segundo orden (Oswaldo} Chateubriand)

Es bien conocido el argumento de Frege en contra de que los números son propiedades de objetos físicos. Éste se enfoca en mostrar que las propiedades numéricas no son distributivas y que, dado un objeto, no siempre es posible determinar su número. Esto llevó a Frege a decir que las atribuciones numéricas son hechas a conceptos y no a cosas, es decir, que son conceptos de conceptos (o propiedades de propiedades). Tomando este hecho como punto de partida, Oswaldo Chateubriand argumenta a favor de que los números son propiedades de segundo orden, es decir, propiedades de propiedades.

Desde luego, la visión extensional que Frege tiene de las propiedades y la insistencia de que (dentro de una ontología dividida de 
manera exhaustiva entre objetos y conceptos) los números son objetos deriva en que la postura de Frege no se considere una teoría de los números como propiedades. Sin embargo, retomando la propuesta del autor, es fácil ver que, si eliminamos la idea de considerar el número $n$ como la extensión del concepto ser equinumeroso con $n$, y nos quedamos sólo con el concepto (ser equinumeroso con $n$ ), la teoría de Frege pasa a ser una teoría de los números como propiedades (pues al eliminar la extensión desaparece el objeto). Esta teoría tiene la virtud de evitar la paradoja de Russell, debido a que ya no es necesaria la famosa Ley básica V que esencialmente dice que, si dos conceptos son coextensionales, entonces hay dos objetos (uno para cada concepto) que son iguales. Es evidente que esta forma de resolver el problema de la paradoja no sería satisfactoria para Frege, entre otras razones, porque su idea de concepto es extensional, por lo que ni siquiera tiene sentido hablar de quitar la extensión y quedarnos con el concepto. También es importante señalar que, como el mismo Chateubriand reconoce, esta teoría fregeana de los números como propiedades tiene sus propios problemas (p. 126).

Otra de las objeciones comunes en contra de un realismo que presente a los números como propiedades proviene de un prejuicio de carácter lingüístico (Hofweber 2000, 2005). Existe una idea generalizada de que los objetos desempeñan el papel nuclear en el sujeto de una oración. Es cierto que paradigmáticamente los objetos hacen las veces de los individuos por los que están los términos singulares que constituyen al sujeto, pero esto no excluye que las propiedades puedan desempeñar este papel, ¿acaso no predicamos también sobre propiedades? Como bien señala Chateubriand, podemos tener una teoría de las propiedades como objetos, es decir, como individuos. La teoría de números tiene los números como individuos; la teoría de conjuntos tiene los conjuntos como individuos; y podemos tener una teoría de funciones donde las funciones son los individuos. Incluso en el lenguaje cotidiano hay expresiones que están por propiedades y que aparecen en los enunciados como individuos. Por ejemplo, expresiones como "El azul es mi color favorito" o "La longitud es una forma de medida". Además, existe también la posibilidad de nominalizar las propiedades. La conclusión es que, el hecho de que los números sean propiedades y aparezcan como individuos en los enunciados aritméticos no representa ningún problema. Simplemente podría generar alguna tensión con cierto paradigma lingüístico en el que hay una correspondencia entre los objetos y los términos singulares, y de forma paralela, entre las propiedades y los determinantes o modificadores. 
Otro problema que discute Chateubriand es que hablar de propiedades de propiedades nos conduce a un sistema de jerarquías, y cuando empezamos a hablar de jerarquías no sabemos dónde parar. Sin embargo, una jerarquía puede generalizarse de muchas maneras que ontológicamente tienen sentido. De hecho (aunque Chateubrian no lo menciona) existen teorías de propiedades que no implican la existencia de jerarquías, por ejemplo, las llamadas type-free (véanse Menzel 1986; Orilia 1991).

En su contribución, Oswaldo Chateubriand parece mostrar satisfactoriamente que una teoría de los números como propiedades puede proporcionar respuestas simples a una gran parte de las objeciones que tradicionalmente se les han presentado a este tipo de explicaciones.

\subsection{Relaciones euclidianas de equinumerosidad (Thomas Sautter)}

De acuerdo con Thomas Sautter, los resultados presentados en este artículo sugieren que abdicar de la relación cantoriana de equinumerosidad en favor de una relación euclidiana, que respete el principio de que el todo es mayor que cualquiera de sus partes propias, nos quita más de lo que estamos dispuestos a ceder en pro del sentido común. El autor pretende mostrar que, en el terreno de las intuiciones, una relación de equinumerosidad cantoriana pierde frente a una relación de equinumerosidad euclidiana al violar nuestra intuición de que el todo es mayor que sus partes. Cuando medimos conjuntos desde un marco cantoriano, obtenemos resultados que parecen contraintuitivos, por ejemplo, que los enteros negativos tienen la misma cardinalidad que el conjunto completo de los enteros, o que el intervalo $(0,1)$ contiene la misma cantidad de puntos que la recta de los números reales. Pese a esto, una relación euclidiana de equinumerosidad elimina la posibilidad de tener una aritmética tan simple y bien determinada como la aritmética de cardinales de Cantor.

La presente estrategia consiste en proponer varias relaciones euclidianas de equinumerosidad. La existencia de una biyección entre dos conjuntos es una condición necesaria pero no suficiente para determinar que éstos son equinumerosos. A partir de la propuesta de una relación en la que se adopta este requisito de la manera más fuerte posible, se proponen otras relaciones que resultan del sucesivo debilitamiento de las anteriores. La idea es que la relación cantoriana es demasiado liberal: la simple existencia de una biyección entre conjuntos los califica como equinumerosos. La relación euclidiana tendría que ser más estricta en este sentido. Por ejemplo, en un conjunto fi- 
nito la relación de equinumerosidad siempre es euclidiana (cualquier subconjunto propio tendrá menos elementos que el total). En este caso, se tiene que se cumple la condición de que para cualquier función inyectiva $f$ entre dos conjuntos equinumerosos, $f$ es también suprayectiva (esta condición no se cumple de manera general para conjuntos infinitos dentro de la aritmética cantoriana). Lo que resulta de este ejercicio es un ejemplo de la tensión que puede darse en matemáticas entre nuestras intuiciones más naturales y los beneficios que provee la práctica matemática.

\subsection{Gödel versus Hilbert y su concepción simbólica del conocimiento matemático (Sérgio Schultz)}

Como es bien sabido, para Hilbert el conocimiento matemático era esencialmente conocimiento simbólico. Sérgio Shultz defiende la idea de que Gödel adoptó esta concepción simbólica de la matemática, de un modo que esto no excluyera su platonismo. Según el autor, la tensión entre dicho simbolismo y el platonismo surge únicamente cuando pensamos a los símbolos como el asunto del que trata la matemática. Para lograr este contraste, Schultz parte de las concepciones de Gödel que se desarrollan a partir de los años treinta, hasta el platonismo que comienza a surgir a mediados de los años cuarenta. La estrecha conexión entre la obra lógico-formal de Gödel y sus preocupaciones marcadamente filosóficas acerca de la fundamentación y la naturaleza del conocimiento matemático sugiere que las concepciones filosóficas de Hilbert tienen una mayor importancia para la ontología y epistemología goedeliana de lo que se ha reconocido en la literatura.

El núcleo del argumento es que el programa de Hilbert representa no solamente el marco lógico-formal en el cual se inserta la obra lógica de Gödel, sino también el marco conceptual previo donde se sitúan y, a partir del cual, se desarrollan las concepciones goedelianas. Respecto al marco lógico-formal, puede que no haya duda de que se da dicha coincidencia. Pero queda pendiente la clarificación de lo que Schultz está tomando como "el marco conceptual". La estrategia del autor es realizar una interpretación de ambos sistemas matemáticos y filosóficos para destacar el espacio de concurrencia. De este modo, es necesario presentar cada uno de ellos de acuerdo con este objetivo.

Por el lado de Hilbert, Schultz sostiene que el formalismo hilbertiano es un formalismo metodológico. Desde una perspectiva fundacional, la matemática transfinita no tiene contenido y el platonismo es inaceptable. La tesis sustancial que subyace al formalismo hilbertiano es que el acceso y el conocimiento de los entes matemáticos se 
obtienen por medio de símbolos. La prueba finitaria de consistencia mostraría la corrección del acceso simbólico propio de las teorías matemáticas y con esto, reivindicaría la concepción simbólica. Por el otro lado, Schultz señala que Gödel adoptó los trazos generales de una concepción simbólica de la matemática como el legado de Hilbert.

Quizá muchos concordaríamos en que para Gödel el problema de los fundamentos era comprendido en términos hilbertianos, en el sentido de que hay que formalizar la teoría de números, y, por medio de la búsqueda de una prueba de consistencia, dar una respuesta al problema fundacional. Lo que resultaría más controversial es la afirmación de Schultz de que esto tenía como finalidad garantizar las suposiciones platónicas de Gödel que, desde un punto de vista fundacional, no pueden ser aceptadas sin justificación.

En conclusión, el desarrollo de las concepciones de Gödel en los años treinta marca la transición de una perspectiva filosófica de los fundamentos de la matemática heredada de Hilbert hacia el platonismo ontológico y epistemológico que será defendido a partir de la mitad de los años cuarenta y hasta el final de su vida. La concepción simbólica hilbertiana involucra un anti-platonismo fundacional que es adoptado por Gödel. No obstante, esta posición es compatible con el platonismo ontológico, en especial, con la tesis de que la matemática transfinita tiene contenido.

Es decir, pareciera que los teoremas de incompletud fueron la bifurcación de un marco conceptual compartido por Hilbert y Gödel. Sin embargo, vale la pena mencionar que en esta exégesis no se están considerando otras ideas filosóficas que ambos autores defendieron de forma tangencial a la teoría de la prueba y a los teoremas de incompletud, respectivamente. Por ejemplo, la idea de Hilbert de que la matemática es completa, en contraste con la idea de Gödel de que, o bien, la matemática es incompleta, es decir, la mente humana es infinitamente superior a cualquier máquina finita, o bien, existen problemas diofánticos absolutamente irresolubles (Gödel 1951).

\subsection{A vueltas con la intuición y el conocimiento matemático}

(Concha Martínez Vidal)

En su aportación a esta colección, Concha Martínez Vidal explora el tema de la intuición y su papel en las teorías de Gödel, Parsons y Bealer. El objetivo es aclarar esta noción, muy discutida en la tradición filosófica, y explorar posibilidades para interpretarla desde el naturalismo. Así, la autora nos presenta una explicación de qué es y cómo fundamenta la intuición el conocimiento matemático 
persiguiendo la metáfora según la cual la intuición sería análoga a la percepción. Además, se presenta la caracterización que Bealer hace de las intuiciones - en tanto que actitudes proposicionales irreductibles y vinculadas a la posesión de conceptos- así como su concepción del tipo de fundamentación que así entendidas proporcionarían para el conocimiento a priori en general.

Como bien lo señala Martínez Vidal, una vez que se asume que la intuición puede ser un elemento fundamental en la justificación del conocimiento matemático, el problema que se plantea es esbozar una concepción de la intuición que dé cuenta de cómo es posible ese tipo de conocimiento sin postular una facultad misteriosa y oscura, que resulte muy diferente de fuentes de conocimiento más familiares, como la percepción, la introspección, la memoria o el testimonio. La tarea es revisar las propuestas de Gödel, Parsons y Bealer desde esta perspectiva.

Para Gödel, la intuición matemática resulta una fuente confiable de conocimiento, no en el sentido de que, si intuimos $p$, entonces $p$ es el caso, sino en el sentido de que en ciertos dominios y siempre que se cultive adecuadamente, la intuición tiende a conducirnos al genuino conocimiento matemático. Por ejemplo, cuando intuimos la verdad de un axioma de la teoría de conjuntos, algo ha de sernos dado en esa intuición. Ese algo serían los objetos y conceptos de la teoría de conjuntos que figuran en ese axioma. Por su parte, para Parsons, la intuición es primariamente intuición de (intuition of) objetos y por tanto particular. Lo esencial de la aritmética es la estructura que los axiomas de Peano caracterizan. Un número no es más que un lugar en esa estructura. Parsons consigue dar cuenta a partir de la intuición de objetos de cómo nos son dados los números y de la noción de sucesión, pero difícilmente puede dar cuenta, según él mismo indica, de la idea de iteración indefinida o infinito.

Finalmente, está la postura de Bealer. Él es, como Martínez Vidal dice, un racionalista moderado que considera que la intuición a priori es una fuente de evidencia básica lo mismo que la percepción. Bealer vincula la intuición a la posesión de conceptos y no al contrario. Las objeciones de Martínez Vidal a esta propuesta son, a) que no explica por qué las intuiciones a priori son fuente de evidencia básica; y b) que no justifica que la intuición no sea reducible a otras actitudes proposicionales como la creencia.

La autora muestra que ninguna de las propuestas analizadas arroja resultados demasiado esperanzadores para la intuición. Sin embargo, las objeciones contra Bealer parecen estar lejos de ser concluyentes. 
Respecto a a), tenemos que para Bealer la intuición es fuente de evidencia básica debido a su confiabilidad (de manera análoga a la percepción), pues éstas son derivadas de la posesión de conceptos debido a que hay un vínculo modal entre ellas y la verdad. En consecuencia, tenemos que en la mayoría de los casos estas intuiciones son correctas. La objeción b) suena un tanto extraña; no hay razón para pensar que la afirmación de que las intuiciones sean derivadas de la posesión de conceptos, implique que éstas no son (o son) atómicas.

Se podría decir que la propuesta de Bealer sería una alternativa que da a las intuiciones el papel fundamental pretendido. Sin embargo, se le concede a la autora que, si se pretenden utilizar las intuiciones para resolver la cuestión de cuál, entre dos conceptos semánticamente estables y poseídos con estabilidad a priori, por parte del sujeto es el correcto, aún queda trabajo por hacer.

\section{Parte III: práctica matemática}

En la tercera y última parte de la compilación se recogen artículos de José Ferreirós, Abel Lasalle Casanave y José M. Sagüillo. Esta parte se encuentra más dirigida a la cuestión epistémica de la certeza aritmética desde la perspectiva de la práctica matemática. Lo que se considera en esta sección como práctica aritmética es amplio y va desde la práctica de contar, hasta actividades matemáticas más complejas que involucran un importante papel epistémico de mecanismos como la intuición y el manejo de simbolismos.

\section{1. Sobre la certeza de la aritmética (José Ferreirós)}

La primera contribución de esta parte es una muy interesante propuesta de José Ferreirós en la cual señala la distinción entre aritmética básica y la(s) teoría(s) de números. La aritmética básica está fundada en última instancia en las prácticas de contar y formalizada en la aritmética de Peano. La(s) teoría(s) de números, en contraste, surgen al examinar propiedades particulares de números naturales. Dentro de estas teorías se incluye el estudio de los modelos de la aritmética de Peano, a saber, la teoría lógica de números. En su artículo, Ferreirós da una respuesta aparentemente satisfactoria al problema epistémico de cómo se adquiere el conocimiento aritmético. Sin embargo, dicha respuesta funciona únicamente para lo que él llama aritmética básica. De acuerdo con el autor, ésta goza de certeza, pero no por ser a priori, sino porque reconocemos la verdad de los principios de Peano en relación con los números de contar; mientras que, respecto a la teoría de números, dado que sus métodos 
pueden exceder la aritmética básica, la certeza (si la hay) no podrá estar fundada en las mismas prácticas. Ferreirós hace énfasis en que afirmar certeza en el conocimiento aritmético no implica en absoluto afirmar su analiticidad ni defender la dicotomía entre verdades empíricas y de razón. Pero lo que sí implica esta tesis es un limitante claro a las nociones pluralistas acerca de las matemáticas.

La clave del resultado epistémico presentado es que el conocimiento matemático se constituye sobre una red de prácticas y de estados cognoscitivos. Red que en último término es enlazada con prácticas y técnicas concretas como la de contar y medir. El autor define aritmética básica como el estudio de las propiedades fundamentales y generales de los números naturales; un cuerpo de teoría que queda bien sistematizado por los axiomas de Peano. Por su parte, una teoría de números es un dominio teórico mucho más amplio, que se origina a partir del estudio de las propiedades particulares de ciertos tipos de números naturales, por ejemplo, los primos. De este modo, la "teoría" de números es una disciplina, no un cuerpo de teoría bien definido; es más bien un complejo de prácticas matemáticas y de cuerpos teóricos asociados, en plural.

La tesis defendida es que los axiomas de Peano son verdaderos de los números de contar. Por medio de la reflexión sobre la práctica de contar y los numerales, advertimos que dichos axiomas son enunciados correctos, verdaderos. El número de contar no es otra cosa que un ingrediente lingüístico o simbólico dentro de un proceso, dentro de una práctica (técnica) razonablemente bien definida: los números de contar se conciben como producto de un proceso de conteo. El sistema de Peano se construye con sólo un cálculo lógico elemental y una noción de clase o conjunto.

La pregunta que se puede responder desde la mencionada distinción es la de cómo puede argumentarse la certeza del conocimiento aritmético, y en relación con el sistema PA de primer orden, cuando es bien conocido que este sistema no puede definir o caracterizar una única estructura.

Es bien sabido que junto al modelo estándar o modelo pretendido de PA, que podríamos describir como la estructura conjuntista que responde a nuestra concepción informal de la totalidad de los números naturales, existe una infinidad de modelos no estándar (numerables y no numerables). Si no podemos caracterizar axiomáticamente, de manera unívoca, la estructura $N=\langle\mathbb{N},+, *\rangle$, si nuestros mejores sistemas lógico-matemáticos sólo alcanzan a determinar una pluralidad de modelos de la aritmética, ¿no habrá que abrazar el 
pluralismo también en este nivel? Ferreirós se pregunta si será esto el fin de toda aspiración a la certeza del conocimiento aritmético.

La cuestión es que los modelos no estándar pertenecen a la Teoría de modelos, que estudia las relaciones entre estructuras conjuntistas, de un lado, y sistemas lógicos-formales, del otro, no pertenecen a la aritmética misma. Igual que se ha dejado otras ramas de la Teoría de números fuera de la tesis de certeza defendida, lo mismo vale para la teoría lógica de números, que no consideramos cierta, sino parte de una matemática avanzada. Como aparecen las cosas una vez que sumergimos la aritmética básica vía PA, en el universo de los modelos conjuntistas, no nos informa acerca de la naturaleza del conocimiento aritmético básico: nos informa acerca de la riqueza del universo conjuntista y modelista, el cual surge de la aceptación de una serie de hipótesis. Nuestro conocimiento numérico básico emerge en la perspectiva bottom-up que va de las prácticas más elementales a las más avanzadas.

En conclusión, muchas de las objeciones que surgen en contra de una tesis que afirme la certeza aritmética, en realidad conciernen a proposiciones que no corresponden a la aritmética misma (a la aritmética básica). A pesar de la claridad del argumento, es de notar la ausencia de ejemplos concretos que ayuden a ilustrar la distinción. La idea parece ser que tenemos certeza acerca de enunciados como " $2+2=4 "$ en virtud de nuestras prácticas de contar, pero esto no tiene una implicación epistémica inmediata respecto a enunciados pertenecientes a alguna teoría de números. Esto es, enunciados aritméticos de teorías correspondientes a modelos no-estándar o enunciados del tipo "Existe una cantidad infinita de números primos". Para un lector que no sea experto en el tema sería de gran utilidad contar con ejemplos que le permitan comprender con mayor profundidad la distinción (crucial para los fines del autor) entre aritmética y teoría(s) de números.

\subsection{Conocimiento simbólico y aritmética en Hilbert (Abel Lasalle Casanave)}

En su aportación a la compilación, Abel Lasalle Casanave considera algunos aspectos del programa de Hilbert en términos de la distinción entre conocimiento intuitivo y simbólico. En este marco, la aritmética finitaria sería una variante de conocimiento simbólico sucedánea del conocimiento intuitivo. Por su parte, la teoría de números, tras la formalización y la justificación proporcionada por una prueba de consistencia, sería conocimiento simbólico formal. El artículo es com- 
parable con algunos otros trabajos presentados en esta compilación; en particular con el trabajo de José Ferreirós y con el de Sérgio Schultz. Respecto al primero, Lasalle Casanave concede certeza únicamente para la aritmética finitaria. En contraste, para Ferreirós, ésta se extiende para toda la aritmética básica formalizada en la aritmética de Peano, incluyendo entonces el principio de inducción. Respecto al trabajo de Sérgio Schultz, parece que ambos autores coinciden en que el formalismo de Hilbert era meramente metodológico. Recordemos que Schultz menciona que éste es compatible incluso con un platonismo como el de Gödel. Lasalle Casanave toma esta misma suposición para obtener un resultado epistémico respecto al conocimiento simbólico.

Para presentar su tesis el autor retoma algunas distinciones clásicas del sistema hilbertiano. En particular, las dualidades ideal-real; finitotransfinito; concreto-abstracto; con contenido (o contentual)-sin contenido; no problemático-problemático. A partir de estas dualidades se puede distinguir entre dos tipos de formalismo. Por un lado, tenemos el formalismo moderado: una parte de la matemática tiene significado - la contentual, finitaria, intuitiva, real, concreta, y no problemática; la otra parte es manipulación simbólica (sin significado) - la no contentual, transfinita, formal, ideal, abstracta y problemática. Por otro lado, tenemos el formalismo extremo, que se limita a ignorar los primeros miembros de las dualidades mencionadas, declarando pura y simplemente que la matemática es manipulación de símbolos sin significado o contenido.

Los primeros términos de las dualidades caracterizan una parte de la matemática como confiable. Los segundos describen otra parte de la matemática como no confiable. La matemática cuestionada por meramente simbólica se justifica a través de una demostración de consistencia. Pero de aquí no se sigue que si el contenido no es intuitivo o concreto se trate sin más de sin-contenido. Para Hilbert, hay contenido transfinito o abstracto, hay proposiciones reales transfinitas, no solamente finitarias, etc. Pero obviamente no hay proposiciones transfinitas intuitivas: lo intuitivo está restricto al punto de vista finito.

Desde el punto de vista finito, las que corresponden a los segundos términos de las dualidades no son afirmaciones confiables y la teoría que las incluya debe ser demostrada como consistente. Se deben también formalizar las operaciones lógicas y las leyes que gobiernan su uso para cumplir la tarea de demostrar consistencia. Según la perspectiva de Lasalle Casanave, ésa es la teoría formal (en sentido puramente sintáctico) de números, en la cual el contenido es rempla- 
zado por manipulación simbólica, y que de esa manera, se constituye en objeto de demostración de consistencia por métodos finitarios.

Respecto a la cuestión epistémica, el autor explica que por la vía simbólica se introducen conceptos y métodos sin contrapartida intuitiva, cuya finalidad es alcanzar el conocimiento que podríamos alcanzar también por procedimientos intuitivos, pero cuya ejecución sería eventualmente muy compleja. Finalmente, reconocemos una tercera variante de conocimiento simbólico como conocimiento de estructuras, formas o relaciones, en cuyo caso, hablamos de conocimiento simbólico como conocimiento formal.

Abel Lasalle concluye que la ontología más adecuada para las ideas de Hilbert sería una de la cual los objetos matemáticos usualmente asumidos no expresasen nada más que nuestra necesidad epistemológica de suponerlos para alcanzar conocimiento (formal) acerca de sistemas de relaciones cuyo acceso es exclusivamente simbólico. Usando terminología moderna, Lasalle Casanave parece considerar a Hilbert como un nominalista hermenéutico: un nominalista que no se compromete con la no existencia de los objetos matemáticos (Burgess y Rosen 1997). Pero, como ocurre cada vez que nos centramos en una postura metafísica, esta posición tiene diversas consecuencias filosóficas, muchas de las cuales derivarán en objeciones. Es difícil saber si Hilbert se hubiera asumido a sí mismo como un nominalista hermenéutico, como parecería sugerirlo Lasalle Casanave.

4.3. Números y proposiciones numéricas en las formalizaciones de la aritmética de Peano, Gödel y Whitehead-Russell (José M. Saguiillo)

En el último trabajo de la compilación, José M. Sagüillo analiza comparativamente tres formalizaciones de la aritmética. Con este propósito en mente, el autor señala dos nociones que a menudo se confunden entre sí. Por un lado, está el dominio de investigación de una ciencia, esto es, su asunto o su género. Éste representa el compromiso ontológico común de todos los que la practican. Por el otro, está el universo de discurso de formalización de una ciencia. Éste se da en un lenguaje formal y representa el compromiso ontológico de las personas que proponen o suscriben dicha formalización. El universo del discurso establecido en una discusión científica dada, puede o no coincidir en extensión con el dominio de la ciencia que se considera. Más aún, una y la misma ciencia con un dominio fijo de investigación puede formalizarse en diferentes lenguajes de diferentes discursos con 
diferentes participantes o grupos y presuponiendo diferentes universos de discurso.

En la formalización de la aritmética de Peano (AP), el dominio es una clase restricta de individuos, mientras que el universo del discurso es la clase irrestricta de todos los individuos. En la formalización de la aritmética de Gödel (AG), el dominio es la clase restricta de individuos como en la formalización de Peano, pero el universo del discurso coincide en extensión con el dominio. En la formalización de Whitehead-Russell (W-R) el dominio es una clase de nociones lógicas en el sentido de Tarski, las cuales no necesariamente son individuos sino ciertas clases de clases de individuos, mientras que el universo del discurso se basa en la clase irrestricta de los individuos como ocurre en la formalización de Peano.

El artículo resulta sumamente enriquecedor, pues además de mostrar la distinción entre universos que el autor propone, aporta claridad al entendimiento vía el contraste de los sistemas acerca de la aritmética de Peano, Gödel y Russell-Whitehead. La distinción entre dominio de discurso de formalización y dominio de investigación provee una buena herramienta teórica para mirar desde una perspectiva filosófica hacia diferentes ramas de la matemática. Por ejemplo, esta distinción resulta muy apropiada cuando tenemos en mente la teoría de conjuntos, que con frecuencia es identificada como el dominio de investigación (el tema) del que tratan múltiples ramas de la matemática. Siendo que lo único que es claro, es que la teoría de conjuntos contribuye a estas ramas con un dominio de discurso de formalización. Quizá valdría la pena enriquecer este trabajo con una explicación sobre la relación entre el dominio de investigación y el dominio de discurso de formalización de una ciencia, así como la utilidad o relevancia filosófica de la distinción que ha resultado expuesta.

El árbol de los números es una compilación de artículos que presenta, de forma exhaustiva y a la vez interesante, debates contemporáneos en filosofía de la mente, filosofía de las matemáticas y lógica relacionados con la noción de número. Los artículos desarrollan el tema de los números enteros no negativos desde diferentes perspectivas filosóficas que, sin lugar a dudas, resultan enriquecedoras, aunque, como suele ocurrir en filosofía, no están completamente exentas de objeciones. 


\section{BIBLIOGRAFÍA}

Balaguer, M., 1998, Platonism and Anti-Platonism in Mathematics, Oxford University Press, Nueva York.

Bueno, O., 2017, "Modal Epistemology and Mathematical Epistemology", en R. Fischer y F. León (comps.), Modal Epistemology After Rationalism, Springer, Dordrecht, pp. 67-83.

Burgess, J.P., 2004, "Mathematics and Bleak House", Philosophia Mathematica, vol. 12, no. 3, pp. 18-36.

Burgess, J.P. y G. Rosen, 1997, A Subject with No Object, Oxford University Press, Nueva York.

Frege, G., 1884, Foundations of Arithmetic, Blackwell, Oxford.

Field, H., 1989, Realism, Mathematics, and Modality, Basil Blackwell, Oxford.

—_ 1980, Science Without Numbers, Princeton University Press, Nueva Jersey.

Gödel, K., 1951, “Algunos teoremas básicos sobre los fundamentos de la matemática y sus implicaciones filosóficas", en Kurt Gödel. Ensayos inéditos, Francisco Rodríguez Consuegra (ed.), 1994, Mondadori, Barcelona.

Hale, B. y C. Wright, 2009, "The Metaontology of Abstraction", en D. Chalmers, R. Wasserman y D. Manley (comps.), Metametaphysics, Oxford University Press, Nueva York.

Hofweber, T., 2005, Number Determiners, Numbers, and Arithmetic, Philosophical Review, vol. 114, no. 2, pp. 179-225.

- 2000, "Quantification and Non-Existent Objects", en A.E. y Tomas Hofweber 2000, Empty Names, Fiction and the Puzzle of the NonExistence, Standford, California, pp. 249-273.

Linnebo, O., 2018, Thin Objects: An Abstractionist Account, Oxford University Press, Nueva York.

Menzel, C., 1986, “A Complete, Type-Free, 'Second-Order' Logic and Its Philosophical Foundations", reporte técnico \#CSLI-86-40, Center for the Study of Language and Information, Stanford University.

Orilia, F., 1991, "Type-Free Property Theory, Exemplification and Russell's Paradox", Notre Dame Journal of Formal Logic, vol. 32, no. 3, pp. 432447.

Yablo, S., 2014, Aboutness, Princeton University Press, Nueva Jersey. , 2010, Things: Papers on Objects, Events, and Properties, Oxford University Press, Nueva York.

Recibido el 23 de julio de 2018; revisado el 8 de enero de 2020; aceptado el 20 de febrero de 2020. 